AIDS, Publish Ahead of Print

DOI: $10.1097 / \mathrm{QAD} .0000000000002732$

\title{
Unveiling the basis of ART-induced osteopenia: the effects of Dolutegravir, Darunavir and Atazanavir on osteogenesis
}

Alessandra Cazzaniga ${ }^{1}$, Roberta Scrimieri ${ }^{1}$, Massimo Galli ${ }^{2}$, Jeanette Maier ${ }^{1}$, and Stefano Rusconi \#

1 General Pathology and 2 Infectious Diseases Unit, Department of Biomedical and Clinical Sciences "Luigi Sacco", Università degli Studi di Milano, Milano, Italy

\# Corresponding authors:

Dipartimento di Scienze Biomediche e Cliniche "Luigi Sacco"

Via G.B. Grassi 74

20157 Milano, Italy

Phone +39-02-50319648

e-mail: jeanette.maier@unimi.it, stefano.rusconi@unimi.it

\section{Running head: The basis of ART-induced osteopenia}

word count: 1708

\section{ABSTRACT}

Objectives: Osteopenia is frequent in HIV infected patients treated with antiretroviral therapy (ART) and has been linked to increased osteoclastogenesis. Little is known about the effects of ART on osteogenesis.

Design: We investigated the effect on human mesenchymal stem cells (hMSC) and osteoblasts of Darunavir and Dolutegravir, the most highly used as anchor drugs within a 3drug regimen, and Atazanavir, which was widely utilized in the past.

Results: We found that Atazanavir and Dolutegravir delay the osteogenic differentiation of hMSC, impair the activity of osteoblasts and inhibit their conversion into osteocytes, while Darunavir exerts no effect.

Conclusions: Atazanavir and Dolutegravir impair osteogenesis. It is essential to early diagnose impaired osteogenesis and to devise effective therapeutic interventions to preserve bone health in ART-treated HIV patients and put it in the context of a correct antiretroviral combination. 


\section{Keywords}

antiretroviral therapy, INSTIs, PIs, mesenchymal stem cell, osteoblast, osteogenesis

\section{Abbreviations}

HIV human immunodeficiency virus

ART Anti-Retroviral Therapy

PIs protease inhibitors

INSTIs integrase strand transfer inhibitors

hMSC human mesenchymal stem cells

RUNX2 Runt-related transcription factor 2

PPARy Peroxisome Proliferator Activated Receptor

BMD bone mineral density

NHOst normal human osteoblasts

ROS Reactive Oxygen Species

DCFH 2'-7'-dichlorofluorescein diacetate

COL1A1 Collagen, type I, alpha 1

BGLAP Bone $\gamma$-carboxyglutamic acid-containing protein

GAPDH Glyceraldehyde-3-Phosphate Dehydrogenase

ALP Alkaline Phosphatase Assay

DMP-1 Dentin matrix acidic phosphoprotein 1

SOST Sclerostin 


\section{INTRODUCTION}

There are more than 30 million people living with human immunodeficiency virus (HIV) infection [1]. About 2/3 receive Anti-Retroviral Therapy (ART), which has significantly reduced HIV-related mortality and increased life expectancy [2]. There are several classes of ART agents that target different stages in HIV lifecycle, i.e. protease inhibitors (PIs), nucleoside and nucleotide reverse-transcriptase inhibitors, non-nucleoside reversetranscriptase inhibitors, entry inhibitors and integrase strand transfer inhibitors (INSTIs) [3]. Among others, Atazanavir and Darunavir are PIs and act by inhibiting aspartyl HIV protease and interfering with the formation of mature viral particles [4]. Dolutegravir belongs to the INSTIs class, binds viral integrase and prevents the incorporation of viral DNA into the host T cell chromosomes [5]. While INSTIs are generally well-tolerated [6], PIs have side effects such as lipoatrophy, cardiovascular disease, metabolic disorders and osteopenia [7].

HIV infected patients seem to have three times higher risk of osteoporosis, osteopenia and fragility fractures than uninfected individuals [8,9]. Indeed, the HIV P55-Gag protein negatively interferes with osteogenic differentiation of human mesenchymal stem cells (hMSC) [10], while the HIV-Rev protein seems to be pro-osteogenic, because it upregulates Runt-related transcription factor 2 (RUNX2), the master regulator of osteogenic differentiation [10]. However, HIV-Rev increases the expression of Peroxisome Proliferator Activated Receptor (PPAR)y, the main driver of adipogenesis [10]. Moreover, circulating HIV-infected B lymphocytes overexpress RANKL and downregulate osteoprotegerin with the consequent enhancement of osteoclast differentiation [7,11] and the PI Atazanavir stimulates osteoclastogenesis in vivo and in vitro [11,12].

Several studies demonstrate that the combination of HIV-infection and ART augments the risk of osteopenia and the amount of the abdominal visceral fat [1,4]. Moreover, patients receiving an ART including PIs present a higher risk of osteopenia than those using other therapeutic regimens [13]. In particular, the decrease of bone mineral density (BMD) caused by PIs seems to be accelerated during the first couple of years of the treatment, while it is stabilized in long term even if the risk of fracture remains high [14].

While osteoclasts activation is well documented in HIV-infected patients treated with ART, less is known about the effects of ART on osteoblastic activity. Osteoblasts derive from hMSC, multipotent stem cells that can differentiate in osteoblasts, chondrocytes or adipocytes in response to specific environmental stimuli [15]. Some studies have reported the inhibition of osteogenic differentiation in hMSC treated with Atazanavir or Lopinavir $[4,16]$. Nowadays, the most highly used anchor drugs within a 3-drug regimen are Darunavir among PIs and Dolutegravir among INSTI. The PI Atazanavir has been widely used until three years ago, when its tolerability in terms of hyperbilirubinemia has decreased drastically its indication. The aim of our study was to evaluate in vitro the effect of these individual drugs from two ART classes, PI and INSTI, on the osteogenic differentiation of hMSC, on the activity of primary human osteoblasts and their terminal differentiation into osteocytes, the most abundant and long-lived cells in human skeleton serving the crucial function of mechanosensors and mechanotransductors [17]. 


\section{MATERIAL AND METHODS}

\section{Cell Culture}

We utilized three strains of hMSC purchased from Lonza cultured according to the manufacturer's instructions (Lonza, Basel, Switzerland) and induced to differentiate by an osteogenic cocktail containing $2 \times 10^{-8} \mathrm{M} 1 \alpha, 25$-Dihydroxyvitamin $\mathrm{D}_{3}, 10 \mathrm{mM} \beta-$ glycerolphosphate and $0.05 \mathrm{mM}$ ascorbic acid (Sigma Aldrich, St. Louis, MO, USA) (osteogenic medium, OM). Three different batches of normal human osteoblasts (NHOst) were purchased from Lonza and maintained as indicated by the manufacturer (Lonza, Basel, Switzerland) [18]. All the cells were used for 3-5 passages. The Alkaline Phosphatase Assay (ALP) fluorimetric Kit (Abcam Cambridge, UK) was utilized to measure ALP in the culture medium following the manufacturer's instructions at $440 \mathrm{~nm}$ (excitation $\lambda=360$ ) using the Glomax Multi-Detection System (Promega, Madison, WI, USA).

To obtain osteocytes, NHOst were spread on collagen type I (Corning Incorporated, New York, USA; final concentration $3 \mathrm{mg} / \mathrm{mL}$ ) and cultured for 14 days [19]. To analyse calcium deposition by hMSC and NHOst, Alizarin Red Staining was performed as described [20].

Cells were exposed to the IC90 concentration, which in vivo inhibits $>90 \%$ of HIV replication and corresponds to $17.4 \mathrm{nM}$ for Atazanavir, $4.1 \mathrm{nM}$ for Darunavir and $145 \mathrm{nM}$ for Dolutegravir. All the results are the mean of at least three independent experiments performed in triplicate.

\section{Real Time PCR}

Total RNA was extracted by the Pure Link RNA Mini Kit (Thermo Fisher Scientific, Waltham, MA, USA) and reverse transcribed using the High-Capacity cDNA Reverse Transcription Kit with RNase inhibitor (Thermo Fisher Scientific, Waltham, MA, USA). Real-time PCR (RT-PCR) was performed three times in triplicate using the CFX96 Touch $^{\mathrm{TM}}$ Real-Time PCR Detection System (Bio-Rad, Hercules, CA, USA) instrument utilizing the TaqMan Gene Expression Assay (Life Technologies, Monza, Italy). The following primers were used: Hs00231692_m1 (RUNX2); Hs00164004_m1, Collagen type I, a1 (COL1A1); Hs01009391_g1 Dentin Matrix Protein (DMP)-1; Hs00228830_m1 Sclerostin (SOST); Hs99999905_m1 Glyceraldehyde-3-Phosphate Dehydrogenase (GAPDH) used as an internal reference gene. Relative changes in gene expression were analysed by the $2^{-\Delta \Delta \mathrm{Ct}}$ method.

\section{Statistical analysis}

Data are reported as media $\pm \mathrm{SD}$. The data were non-parametric and normally distributed and they were analysed using two-way ANOVA (hMSC) and one-way ANOVA (NHOst). The $p$ values deriving from multiple pairwise comparisons were corrected by the Bonferroni method. Statistical significance was defined for $p$-value $<0.05$.

\section{RESULTS}

\section{Osteogenic differentiation after exposure to Atazanavir, Darunavir or Dolutegravir}

hMSC were exposed for various times to Atazanavir, Darunavir or Dolutegravir in the presence or in the absence of an osteogenic cocktail (OM and culture medium, CM, 
respectively). RT-PCR was performed using specific primers for RUNX2, essential for osteoblast differentiation, and COL1A1, coding for collagen $1 \alpha$ [21]. As expected [20,22], RUNX2 and COL1A1 were overexpressed after 3 and 7 days of culture in OM in untreated cell. Atazanavir, Darunavir or Dolutegravir partially inhibited OM-induced expression of $R U N X 2$ and COL1A1 (Figure 1A-B).

We then evaluated whether the differences of gene expression were translated into differences in calcium deposition by staining the cells with Alizarin Red after 7 and 14 days of culture in osteogenic medium with or without the three drugs. The presence of calcium deposits is a biomarker of osteogenic differentiation of hMSC. Figure 1C shows that Atazanavir and Dolutegravir impair calcium deposition, while Darunavir has no effect.

\section{Osteoblast activity after exposure to Atazanavir, Darunavir or Dolutegravir}

NHOst treated with Atazanavir and Dolutegravir for 14 days lost their ability to deposit calcium. Darunavir did not exert any effect (Figure 2A). Accordingly, the release of ALP, which is critical in bone formation [23], was decreased in cells treated with Atazanavir and Dolutegravir, while Darunavir had no effect (Figure 2B).

\section{Transition from osteoblasts in osteocytes after exposure to Atazanavir, Darunavir or Dolutegravir}

After depositing bone matrix, osteoblasts are embedded in this matrix and terminally differentiate into osteocytes [19]. NHOst were cultured in type I collagen gels for 14 days in the presence or not of the drugs and the expression of two osteocytic markers, DMP-1 and SOST $[24,25]$, was analyzed by RT-PCR. DMP-1 is expressed in early embedding osteocytes and is maintained thereafter. SOST is only expressed in deeply-embedded late osteocytes [24]. Controls and cells treated with Darunavir overexpress DMP-1 and SOST, thus indicating their conversion into osteocytes, while cells treated with Atazanavir and Dolutegravir do not (Figure 2C).

\section{DISCUSSION}

ART grants the possibility to reduce viral load to almost undetectable levels and has converted what used to be a fatal disease into a chronic disease, with unprecedented outcomes [26]. However, persistent inflammatory responses associated with HIV infection as well as adverse drug reactions impact on the skeleton, to the point that HIV infection and ART are considered independent risk factors for osteopenia [27,28]. Indeed, ART initiation has been linked to changes of bone mineral density [29]. Now that HIV population begins to age, an increase in bone fractures at an uncharacteristically young age, especially for men, is becoming evident and this trend might worsen as patients shift to an older demographic $[13,30]$.

Because the effects of ART on bone cells is unclear and controversial, we investigated the direct effects of the PIs Atazanavir and Darunavir or the INSTI Dolutegravir on the osteogenic differentiation of hMSC, on the activity of primary osteoblasts and their transition into osteocytes. 
These drugs partially inhibit OM-induced expression of the early markers of osteogenesis RUNX2 and COL1A1. RUNX2 is fundamental in committing MSC to the osteoblast lineage [31]. It triggers the expression of genes coding for bone matrix proteins, among which COL1A1 and some non-collagenous matrix proteins [31]. With the gradual acquisition of a mature phenotype, RUNX2 declines and other transcription factors come into play [31].

The outcome of osteogenic differentiation is the formation of calcium deposits in the extracellular matrix. While Darunavir-treated cells behave like the controls, Dolutegravir and Atazanavir impair the deposition of calcium. Our results are in agreement with a previous paper showing that Atazanavir reduces osteoblast differentiation [16]. Similarly, the inhibition of osteogenic differentiation of hMSC treated with some PIs (i.e Saquinavir, Liponavir and Nelfinavir) was reported [4].

Focusing on mature osteoblasts, Darunavir grants the normal function of these cells, whereas Atazanavir and Dolutegravir inhibit osteoblastic activity. Therefore, our results suggest that ALP, a predictor of bone density, should be routinely measured in ART-treated patients to monitor osteoblastic activity.

Atazanavir and Dolutegravir also prevent the terminal differentiation of osteoblasts into osteocytes, while Darunavir does not. Osteocytes are crucial in bone homeostasis, since they actively orchestrate the activity of osteoblasts and osteoclasts [17]. We hypothesize that a reduced number of osteocytes in Atazanavir- and Dolutegravir-treated patients might subvert cell-to-cell communication in the bone, resulting in altered bone structure.

While Atazanavir is associated with a higher risk of osteoporosis in women [13], at the moment no data are available about the action of Dolutegravir on BMD in patients [32] nor on its effects on bone cells, but no alarming signals have been reported in clinical trials. The $3^{\text {rd }}$ drug - i.e. anchor drug - effect has been reduced by the burden of other nucleoside/nucleotide inhibitors of HIV reverse transcriptase. By the available evidences, INSTI do not seem to impact on the bone health of individuals receiving antiretrovirals.

Since ART exerts adverse effects on bone [13], a better understanding of the mechanisms responsible for skeletal deterioration will be essential to forge effective therapeutic interventions to safeguard all the significant improvements achieved in the long-term health of patients living with HIV/AIDS.

\section{ACKNOWLEDGEMENTS}

SR and JM were funded by DIBIC “Luigi Sacco”, Università di Milano Linea 2.

This work had been partially funded through research grants from ViiV Healthcare S.r.l., Verona, Italy, and Janssen-Cilag S.p.A., Cologno Monzese (MI), Italy.

\section{DECLARATIONS OF INTEREST}

SR has received research grants or personal fees for CME activities or advisory boards from ViiV, MSD, BMS, Gilead, Janssen and Mylan. MG has received research grants or personal fees for CME activities or advisory boards from ViiV, MSD, BMS, Gilead, Janssen, and Abbott. All other authors declare no conflict of interest. 


\section{REFERENCES}

[1] A.N. Ahmad, S.N. Ahmad, N. Ahmad, HIV Infection and Bone Abnormalities, The $\begin{array}{lllll}\text { Open } & \text { Orthopaedics } & \text { Journal. } & 11 & \text { (2017) }\end{array}$ https://doi.org/10.2174/1874325001711010777.

[2] J. Poorolajal, E. Hooshmand, H. Mahjub, N. Esmailnasab, E. Jenabi, Survival rate of AIDS disease and mortality in HIV-infected patients: a meta-analysis, Public Health. 139 (2016) 3-12. https://doi.org/10.1016/j.puhe.2016.05.004.

[3] E.J. Arts, D.J. Hazuda, HIV-1 antiretroviral drug therapy, Cold Spring Harbor Perspectives in Medicine. 2 (2012) a007161-a007161. https://doi.org/10.1101/cshperspect.a007161.

[4] R.G. Jain, J.M. Lenhard, Select HIV protease inhibitors alter bone and fat metabolism ex vivo, Journal of Biological Chemistry. 277 (2002) 19247-19250. https://doi.org/10.1074/jbc.C200069200.

[5] D.J. Hazuda, P. Felock, M. Witmer, A. Wolfe, K. Stillmock, J.A. Grobler, A. Espeseth, L. Gabryelski, W. Schleif, C. Blau, M.D. Miller, Inhibitors of strand transfer that prevent integration and inhibit HIV-1 replication in cells, Science. 287 (2000) 646-650. https://doi.org/10.1126/science.287.5453.646.

[6] C.E. Kandel, S.L. Walmsley, Dolutegravir - A review of the pharmacology, efficacy, and safety in the treatment of HIV, Drug Design, Development and Therapy. 9 (2015) 3547-3555. https://doi.org/10.2147/DDDT.S84850.

[7] K. Titanji, A. Vunnava, A.N. Sheth, C. Delille, J.L. Lennox, S.E. Sanford, A. Foster, A. Knezevic, K.A. Easley, M.N. Weitzmann, I. Ofotokun, Dysregulated B Cell Expression of RANKL and OPG Correlates with Loss of Bone Mineral Density in HIV Infection, PLoS Pathogens. $10 \quad$ (2014) 8-10. https://doi.org/10.1371/journal.ppat.1004497.

[8] L. Battalora, K. Buchacz, C. Armon, E.T. Overton, J. Hammer, P. Patel, J.S. Chmiel, K. Wood, T.J. Bush, J.R. Spear, J.T. Brooks, B. Young, Low bone mineral density and risk of incident fracture in HIV-infected adults, Antiviral Therapy. 21 (2016) 45-54. https://doi.org/10.3851/IMP2979.

[9] T.T. Brown, R.B. Qaqish, Antiretroviral therapy and the prevalence of osteopenia and osteoporosis: A meta-analytic review, Aids. 20 (2006) 2165-2174. https://doi.org/10.1097/QAD.0b013e32801022eb.

[10] E.J. Cotter, H.S.M. Ip, W.G. Powderly, P.P. Doran, Mechanism of HIV protein induced modulation of mesenchymal stem cell osteogenic differentiation, BMC Musculoskeletal Disorders. 9 (2008) 1-12. https://doi.org/10.1186/1471-2474-9-33.

[11] G. Pan, Z. Yang, S.W. Ballinger, J.M. McDonald, Pathogenesis of osteopenia/osteoporosis induced by highly active anti-retroviral therapy for AIDS, Annals of the New York Academy of Sciences. 1068 (2006) 297-308. https://doi.org/10.1196/annals.1346.057. 
[12] M.J.F. Blumer, B. Hausott, C. Schwarzer, A.R. Hayman, J. Stempel, H. Fritsch, Role of tartrate-resistant acid phosphatase (TRAP) in long bone development., Mechanisms of Development. 129 (2012) 162-176. https://doi.org/10.1016/j.mod.2012.04.003.

[13] C.A. Moran, M. Neale Weitzmann, I. Ofotokun, The protease inhibitors and HIVassociated bone loss, Current Opinion in HIV and AIDS. 11 (2016) 333-342. https://doi.org/10.1097/COH.0000000000000260.

[14] H. Hirakawa, H. Gatanaga, H. Ochi, T. Fukuda, S. Sunamura, S. Oka, S. Takeda, S. Sato, Antiretroviral Therapy Containing HIV Protease Inhibitors Enhances Fracture Risk by Impairing Osteoblast Differentiation and Bone Quality, Journal of Infectious Diseases. 215 (2017) 1893-1897. https://doi.org/10.1093/infdis/jix246.

[15] B.M. Abdallah, M. Kassem, Human mesenchymal stem cells: From basic biology to clinical applications, Gene Therapy. $15 \quad$ (2008) 109-116. https://doi.org/10.1038/sj.gt.3303067.

[16] S.J. Hernandez-Vallejo, C. Beaupere, J. Larghero, J. Capeau, C. Lagathu, HIV protease inhibitors induce senescence and alter osteoblastic potential of human bone marrow mesenchymal stem cells: Beneficial effect of pravastatin, Aging Cell. 12 (2013) 955-965. https://doi.org/10.1111/acel.12119.

[17] L. Qin, W. Liu, H. Cao, G. Xiao, Molecular mechanosensors in osteocytes, Bone Research. 8 (2020) 1-24. https://doi.org/10.1038/s41413-020-0099-y.

[18] A. Cazzaniga, S. Castiglioni, J.A.M. Maier, Conditioned media from microvascular endothelial cells cultured in simulated microgravity inhibit osteoblast activity, BioMed Research International. 2014 (2014). https://doi.org/10.1155/2014/857934.

[19] K. Uchihashi, S. Aoki, A. Matsunobu, S. Toda, Osteoblast migration into type I collagen gel and differentiation to osteocyte-like cells within a self-produced mineralized matrix: A novel system for analyzing differentiation from osteoblast to osteocyte, Bone. 52 (2013) 102-110. https://doi.org/10.1016/j.bone.2012.09.001.

[20] S. Castiglioni, V. Romeo, L. Locatelli, A. Cazzaniga, J.A.M. Maier, TRPM7 and MagT1 in the osteogenic differentiation of human mesenchymal stem cells in vitro., Scientific Reports. 8 (2018) 16195. https://doi.org/10.1038/s41598-018-34324-8.

[21] A. Cazzaniga, J.A.M. Maier, S. Castiglioni, Impact of simulated microgravity on human bone stem cells: New hints for space medicine, Biochemical and Biophysical $\begin{array}{llll}\text { Research } & \text { Communications. } & 473 & \text { (2016) }\end{array}$ https://doi.org/10.1016/j.bbrc.2016.03.075.

[22] A. Sargenti, S. Castiglioni, E. Olivi, F. Bianchi, A. Cazzaniga, G. Farruggia, C. Cappadone, L. Merolle, E. Malucelli, C. Ventura, J.A.M. Maier, S. Iotti, Magnesium deprivation potentiates human mesenchymal stem cell transcriptional remodeling, International Journal of Molecular Sciences. $19 \quad$ (2018). https://doi.org/10.3390/ijms19051410.

[23] M. Leidi, F. Dellera, M. Mariotti, J.A.M. Maier, High magnesium inhibits human 
osteoblast differentiation in vitro., Magnesium Research. 24 (2011) 1-6. https://doi.org/10.1684/mrh.2011.0271.

[24] D. Guo, L.F. Bonewald, Advancing our understanding of osteocyte cell biology, Therapeutic Advances in Musculoskeletal Disease. 1 (2009) 87-96. https://doi.org/10.1177/1759720X09341484.

[25] W. Albisetti, L.S. Giarratana, C. Viganò, S. Castiglioni, J.A. Maier, Sclerostin: A novel player regulating bone mass in inflammation?, European Journal of Inflammation. 11 (2013) 345-352. https://doi.org/10.1177/1721727X1301100204.

[26] K. Buchacz, C. Armon, F.J. Palella Jr, R.M. Novak, J. Fuhrer, E. Tedaldi, D. Ward, C. Mayer, L. Battalora, K. Carlson, S. Purinton, M. Durham, J. Li, H.I.V.O.S. (HOPS) Investigators, The HIV Outpatient Study-25 Years of HIV Patient Care and Epidemiologic Research, Open Forum Infectious Diseases. 7 (2020) ofaa123-ofaa123. https://doi.org/10.1093/ofid/ofaa123.

[27] G.A. McComsey, P. Tebas, E. Shane, M.T. Yin, E.T. Overton, J.S. Huang, G.M. Aldrovandi, S.W. Cardoso, J.L. Santana, T.T. Brown, Bone disease in HIV infection: a practical review and recommendations for HIV care providers., Clinical Infectious Diseases : An Official Publication of the Infectious Diseases Society of America. 51 (2010) 937-946. https://doi.org/10.1086/656412.

[28] T.T. Brown, J. Hoy, M. Borderi, G. Guaraldi, B. Renjifo, F. Vescini, M.T. Yin, W.G. Powderly, Recommendations for evaluation and management of bone disease in HIV., Clinical Infectious Diseases: An Official Publication of the Infectious Diseases Society of America. 60 (2015) 1242-1251. https://doi.org/10.1093/cid/civ010.

[29] J. Compston, HIV infection and bone disease, Journal of Internal Medicine. 280 (2016) 350-358. https://doi.org/10.1111/joim.12520.

[30] M.S. Rothman, M.T. Bessesen, HIV infection and osteoporosis: Pathophysiology, diagnosis, and treatment options, Current Osteoporosis Reports. 10 (2012) 270-277. https://doi.org/10.1007/s11914-012-0125-0.

[31] T. Komori, Regulation of bone development and extracellular matrix protein genes by RUNX2., Cell and Tissue Research. 339 (2010) 189-195. https://doi.org/10.1007/s00441-009-0832-8.

[32] P. Tebas, P. Kumar, C. Hicks, C. Granier, B. Wynne, S. Min, K. Pappa, Greater change in bone turnover markers for efavirenz/emtricitabine/tenofovir disoproxil fumarate versus dolutegravir $\mathrm{R}$ abacavir/lamivudine in antiretroviral therapy-naive adults over $144 \quad$ weeks, Aids. $29 \quad$ (2015) 2459-2464. https://doi.org/10.1097/QAD.0000000000000863. 


\section{FIGURE LEGENDS}

Figure 1. Osteogenic differentiation of hMSC in the presence of Atazanavir, Dolutegravir or Darunavir.

The cells were cultured in the presence of the individual drugs with or without the osteogenic cocktail.

(A-B) RT-PCR was performed for RUNX2 (A), COL1A1 (B).

(C) Calcium deposition was assessed by Alizarin Red Staining.

Note: $* p<0.05 ; * * p<0.01 ; * * * p<0.001$.

A
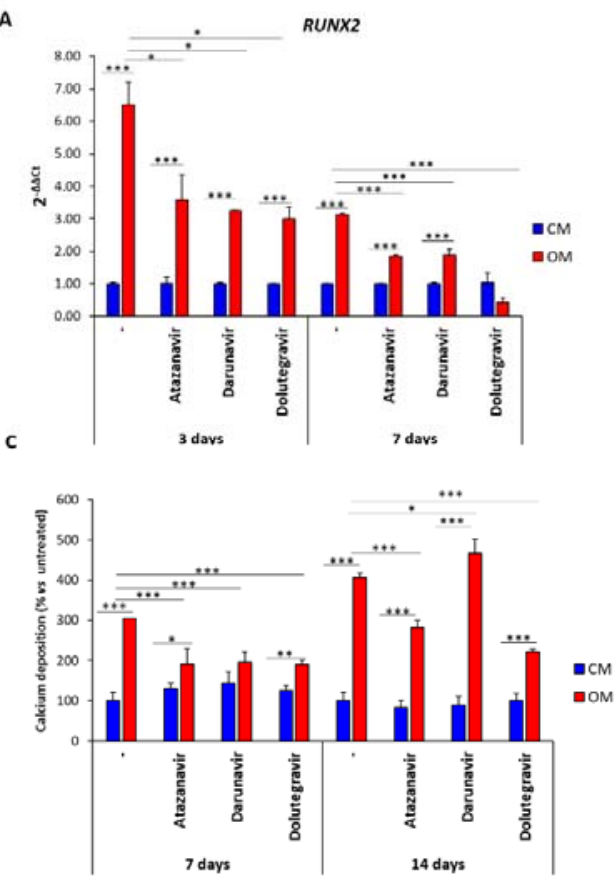

B

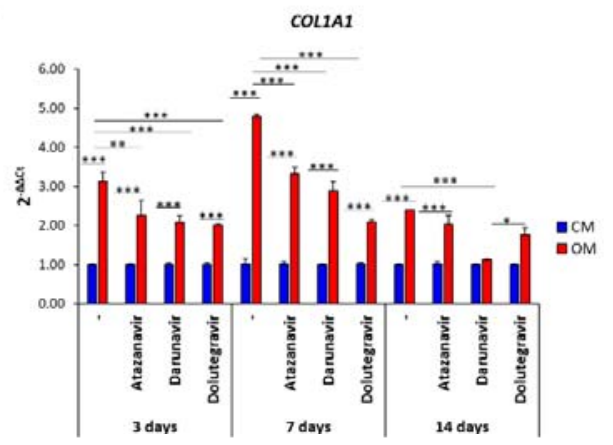


Figure 2. Osteoblast activity and their terminal differentiation into osteocytes in the presence of Atazanavir, Dolutegravir or Darunavir.

NHOST were cultured in the presence of the individual drugs.

A) Calcium deposition was assessed by Alizarin Red Staining.

B) ALP activity was measured using a fluorimetric ALP kit. Data are expressed as percentages of the fold increase of the signal intensity obtained in culture media in the treated vs untreated (-).

C) After culture in collagen gel for 14 days in the presence of the drugs, RT-PCR was performed using primers designed on DMP-1 and SOST sequences.

Note: $* p<0.05 ; * * p<0.01 ; * * * p<0.001$.

A

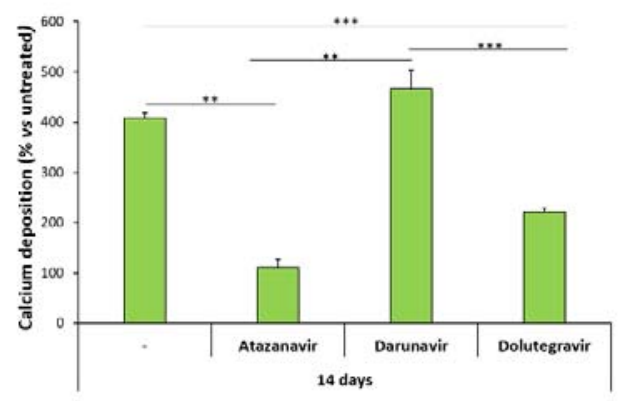

B

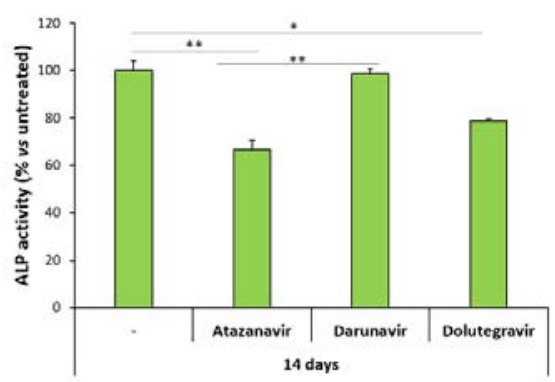

c

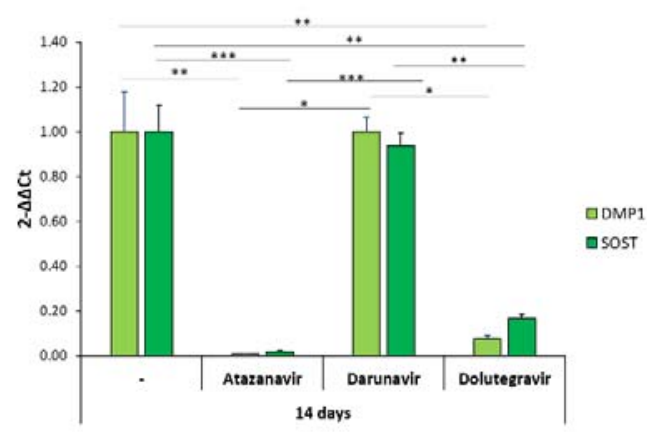

